\title{
Design, energia solar por conversão fotovoltaica e artefato de medição ${ }^{1}$
}

\section{Design, photovoltaic solar energy and measurement artifact}

\author{
Flavia Aparecida Silveira, UFPR.
}

flaasilveira@gmail.com

Aloísio Leoni Schmid, UFPR.

aloisio.schmid@gmail.com

\section{Resumo}

Este estudo aborda a melhoria da integração dos sistemas fotovoltaicos na arquitetura e no design. Para isso, questiona-se como mensurar a variação da energia elétrica produzida pela conversão fotovoltaica da energia solar, considerando a orientação, a inclinação da superfície coletora e a tecnologia de conversão, na cidade de Curitiba, Paraná. Com base na teoria da conversão da energia fotovoltaica, simulações realizadas em estudos anteriores e utilizando características do Design Science Research é desenvolvido um artefato para medição de radiação solar em condições energeticamente sub-ótimas. Para isso são utilizados módulos de silício policristalino, silício amorfo e filme fotovoltaico orgânico como tecnologias de conversão. $\mathrm{O}$ artefato é criado com o intuito de demonstrar possibilidades de aplicação da tecnologia tanto em produtos de design quanto em projetos arquitetônicos.

Palavras-chave: Design Science Research, Energia Fotovoltaica, Artefato

\begin{abstract}
This study addresses the improvement of the integration of photovoltaic systems into architecture and design. The problem question is on how the electric energy production by the photovoltaic conversion is influenced by angle, azimuth of the collecting surface and the conversion technology, in Curitiba, Paraná. Based on the photovoltaic energy conversion theory, as well as simulations carried out in previous studies and using features of Design Science Research, an artifact is developed for measuring solar radiation in energetically suboptimal conditions. For this purpose, polycrystalline silicon, amorphous silicon and organic photovoltaic modules are used as conversion technologies. The artifact is created in order to demonstrate possibilities for applying technology both in design products and in architectural projects.
\end{abstract}

Keywords: Design Science Research, Photovoltaic Energy, Artifact

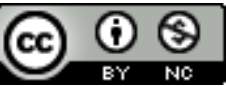

\footnotetext{
1 Este trabalho foi realizado com o apoio da 'Companhia Paranaense de Energia - COPEL' programa de pesquisa e desenvolvimento tecnológico, projeto PD 2866-0470-2017, regulamentado pela ANEEL.

This work has been supported by the 'Companhia Paranaense de Energia - COPEL' research and technological development program, through the PD 2866-0470/2017 project, regulated by ANEEL
} 


\section{Introdução}

Este trabalho está relacionado à energia solar por conversão fotovoltaica e às possíveis aplicações fora das condições ideais de orientação e inclinação, bem como o aproveitamento da reflexão pelo entorno (albedo). Tais fatores podem ser empregados, por exemplo, em telhados na área urbana e no desenvolvimento de produtos que empreguem módulos fotovoltaicos.

Um fator de interferência é o posicionamento dos módulos fotovoltaicos. A combinação ideal de orientação e inclinação proporciona um maior rendimento da instalação fotovoltaica e permite, conhecendo o valor da irradiação solar incidente, o cálculo da energia elétrica que pode ser convertida por um sistema nessas condições. Tal combinação, segundo Villalva e Gazoli (2013 p. 49-60) tem a orientação para a linha do equador e o ângulo de inclinação igual à latitude do local em que o sistema é posicionado. Em Curitiba - Paraná, a orientação ideal é o Norte Verdadeiro com inclinação corresponde à latitude da cidade, aproximadamente $25^{\circ}$.

Ao desviar dessa orientação ideal há um problema sobre como estimar a perda na conversão de energia. Neste sentido, a sequência deste estudo se dá com o desenvolvimento do projeto de um artefato de medição que possibilita entender a interferência do albedo e da inclinação em módulos de silício policristalino, silício amorfo e módulos orgânicos para conversão fotovoltaica.

\section{Radiação direta, difusa e refletida}

A geometria da insolação auxilia no posicionamento dos módulos fotovoltaicos, seja para tornar a captação de energia mais eficiente ou para apresentar a diferença no desempenho da conversão fotovoltaica de acordo com o projeto. Neste sentido, é importante entender como funcionam alguns parâmetros da geometria da insolação, como radiação direta, difusa e albedo e também a irradiação global, que compreende a soma destas. Para cada localidade, ela é medida diariamente e são registrados os totais mensais e anuais.

Nesta perspectiva Frota (2001 p. 54) aponta que a radiação solar "é uma energia eletromagnética, de onda curta, que atinge a Terra após ser parcialmente absorvida pela atmosfera". Assim os fluxos de irradiação solar são determinados a partir de fatores meteorológicos, astronômicos e geográficos. Frota (2001 p. 54) menciona que as quantidades de radiação variam em função da época do ano e da latitude.

A radiação direta é aquela proveniente do sol que atinge diretamente o módulo fotovoltaico. De acordo com Querino (2006), chega à superfície sem sofrer alteração significativa, sendo afetada apenas pela refração proveniente da atmosfera.

Já a radiação difusa é a que chega ao módulo fotovoltaico após passar por outra superfície, como gases, nuvens e afins. Para Querino (2006) a radiação difusa é proveniente de todas as direções, pois é espalhada por gases, gotas d'água e material particulado presentes na atmosfera.

Há ainda uma parcela refletida, depois de atingir a superfície da Terra. O albedo, ou refletância do entorno pode ser entendido como a fração da radiação incidente numa superfície 
que é refletida de modo difuso. Diferente das outras parcelas, o albedo pode ser alterado por decisões do projeto.

\section{Células e módulos fotovoltaicos}

As células fotovoltaicas podem ser compostas por diversos materiais semicondutores. Estes podem ter tamanhos, cores e formas, de acordo com a tecnologia empregada e o desenvolvimento de pesquisas neste campo. Alguns exemplos são as células compostas por silício policristalino, monocristalino ou amorfo. Também podem ser citados como exemplo os módulos fotovoltaicos orgânicos cuja composição pode conter, de acordo com Yamamoto (2014, p.29) polímeros conjugados ou pequenas moléculas conjugadas.

As células fotovoltaicas estão em constante estudo e desenvolvimento. Machado e Miranda (2015, p. 129-136) e Slaoui (2017) apontam que atualmente são encontradas células de primeira, segunda e terceira geração, classificadas de acordo com o modo de produção e os materiais utilizados. Usualmente, as células são agrupadas em módulos fotovoltaicos de acordo com suas características, que podem variar com relação à geração, dimensões e formatos.

$\mathrm{Na}$ primeira geração estão as células compostas por silício monocristalino (mc-SI) ou policristalino (p-Si). Estas foram objeto de estudos consolidados e apresentam maior eficiência de conversão de energia até o momento. A eficiência de conversão varia de acordo com o modo de produção dos módulos, sendo que os módulos monocristalinos apresentam eficiência de conversão de aproximadamente 15 a $25 \%$ e os módulos policristalinos de 14 a $20 \%$. Na Erro! Fonte de referência não encontrada. é possível observar exemplos de células
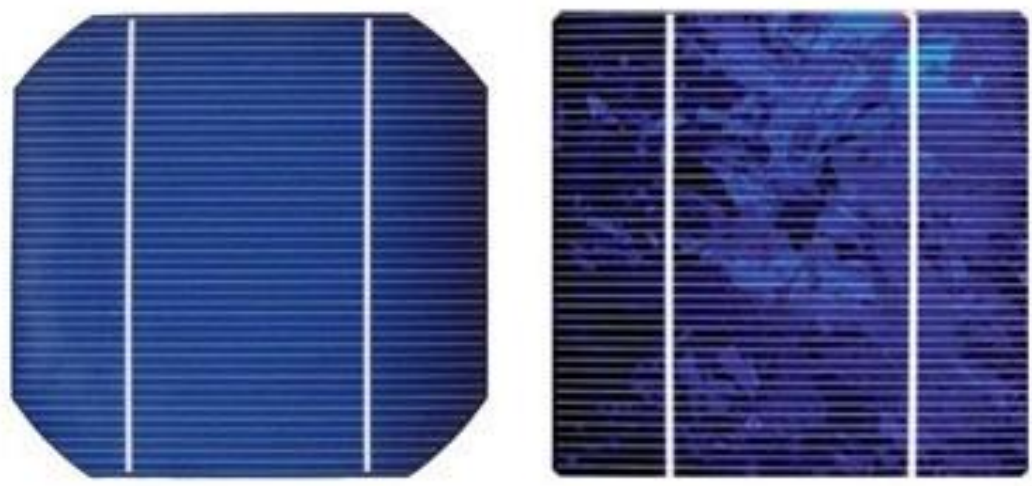

Figura 1 - Célula de Silício Monocristalino à esquerda e célula de Silício Policristalino à direita. Fonte: 1st Sunflower Renewable Energy (2020)

fotovoltaicas de primeira geração. 
Em relação à segunda geração, estão as células de filme fino, constituídas por materiais como o silício amorfo (a-Si) ou arseneto de gálio (GaAs). Estas células apresentam redução na eficiência, que usualmente fica entre 6 e 15\%, dependendo do tipo de material e fabricação. Por apresentarem transparência predefinida, possibilitam uma maior gama de formas e aplicações, como pode ser visualizado no exemplo da Figura 2.

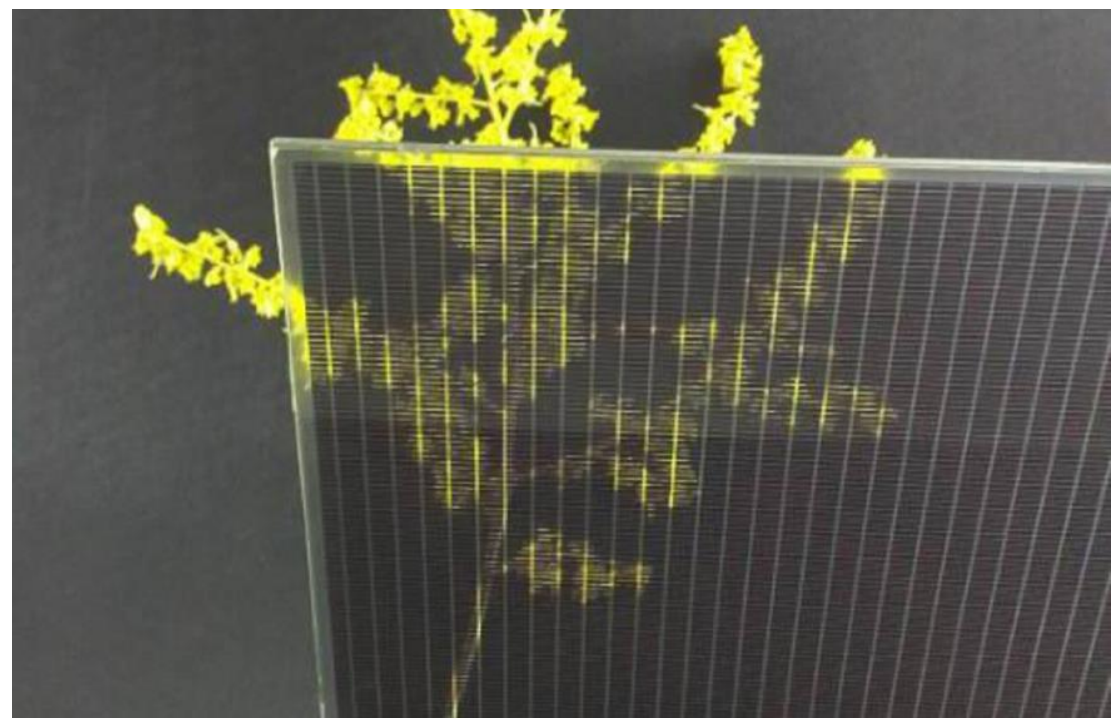

Figura 2 - Módulo Fotovoltaico de Silício Amorfo. Fonte: Hangzhou Amplesun Solar Technology (2020)

Entre as células de terceira geração estão as que utilizam materiais orgânicos, como OPV (organic photovoltaic) e as células sensibilizadas por corantes. As pesquisas nesta área seguem em desenvolvimento para aperfeiçoar os módulos e a comercialização desta tecnologia é realizada por poucos fornecedores. A eficiência de conversão destes módulos varia de acordo com a cor, material e fabricação. Um exemplo de OPV pode ser observado na Figura 3.

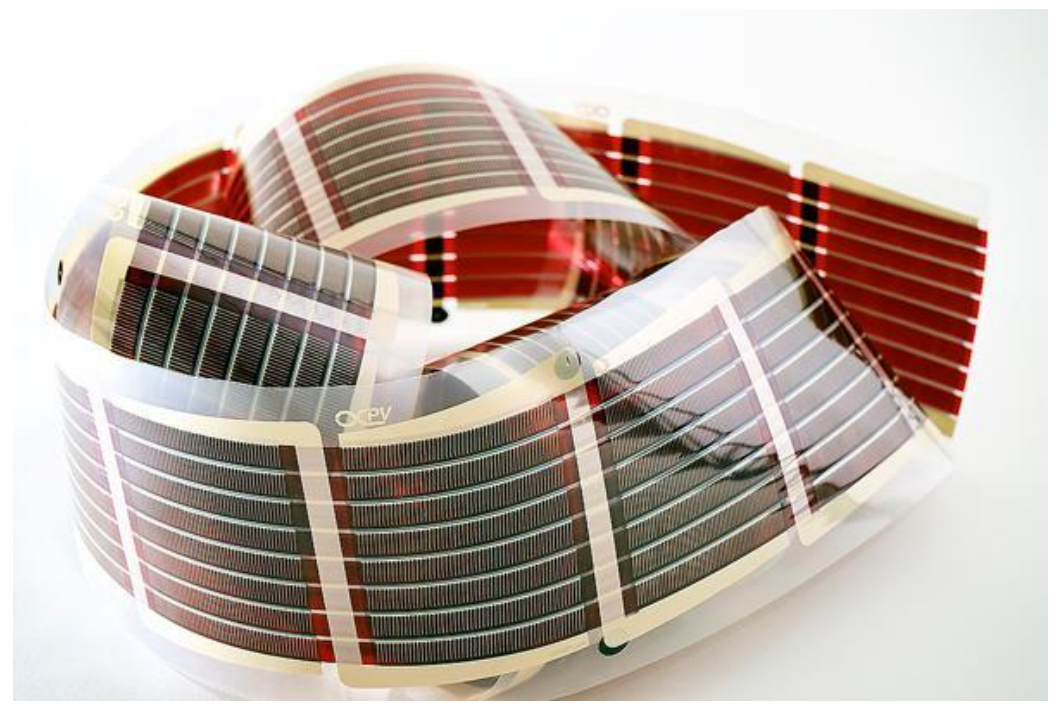

Figura 3 - Módulo fotovoltaico orgânico - OPV. Fonte: Infinity PV (2020) 
A ABNT NBR 10899/2013 define que o módulo fotovoltaico é uma unidade básica formada por um conjunto de células fotovoltaicas, interligadas eletricamente e encapsuladas, com o objetivo de gerar energia elétrica.

Cabe ao design aprimorar os módulos que são utilizados para a conversão da energia solar fotovoltaica, tanto na criação de novos modelos que visam aderência comercial e a possibilidade de utilização de forma integrada à arquitetura (BIPV - Building Integrated Photovoltaics), quanto no desenvolvimento de fatores técnicos como encaixes e conexões que garantam estanqueidade e confiabilidade a transmissão da energia gerada, ou mesmo a possibilidade de uso de outros materiais para melhorar o desempenho dos módulos.

A seleção e o posicionamento dos módulos fotovoltaicos segundo o paradigma da orientação ideal são rotineiros no contexto brasileiro. Contudo, um fator que pode ser decisivo na integração arquitetônica de módulos fotovoltaicos é o albedo, pois este pode auxiliar na compensação da geração de energia de módulos voltados para orientações em condições energeticamente sub-ótimas. Neste ponto vale lembrar que, a exemplo do petróleo, os módulos fotovoltaicos são commodities sujeitas ao comportamento de um oligopólio. Flutuações de preço são esperadas no futuro, o que condiciona a busca por sistemas eficientes, mesmo que não ótimos.

\section{Energia solar fotovoltaica e design}

A conversão fotovoltaica pode ser utilizada de diversas formas, tanto em sistemas integrados à rede de transmissão de energia (on grid), sistemas não integrados (off grid) e também no desenvolvimento de mobiliário urbano e design de produtos. Atualmente existem produtos de diversos segmentos que utilizam módulos fotovoltaicos, desde calculadoras até carros. Estes podem ser considerados como produtos PIPV (product integrated photovoltaics).

Em relação à instalação e à medição da energia solar pela conversão fotovoltaica on grid, Villalva e Gazoli (2013 p.152-210) apontam que os sistemas fotovoltaicos são compostos por conjuntos de módulos e inversores adequados às necessidades do projeto. Algumas dessas necessidades são a tensão de operação na conexão com a rede, o grau de proteção do equipamento, o consumo de energia, a potência de entrada e saída do inversor e seu rendimento e a tensão e frequência de operação.

Os sistemas on grid contam ainda com medidores que registram o consumo e a geração elétrica, inversores, caixas de strings (caixa de conexões), quadro de proteção de corrente contínua e alternada e dispositivos de proteção contra surtos.

Os sistemas off grid podem ser utilizados, segundo Villalva e Gazoli (2013 p. 99-125), em uma infinidade de aplicações, desde aparelhos eletrônicos até sistemas aeroespaciais, passando por veículos e entrega de eletricidade em locais remotos.

Observando estes pontos é possível inferir que o design possa auxiliar tanto na criação de produtos que utilizam a tecnologia fotovoltaica quanto no desenvolvimento da própria tecnologia, tornando-a melhor nos aspectos que tangem o design. 
Para tanto, alguns pontos abordados pelos autores requerem atenção, como a utilização de baterias, os controladores de carga e a utilização ou não de conversores, bem como barreiras a serem transpostas para a utilização desta energia. Santos (2013 p.195) exemplifica que um fator que dificulta a ampliação da utilização da energia solar pela conversão fotovoltaica é o desconhecimento dos arquitetos brasileiros em relação às formas de utilização dos módulos fotovoltaicos, como por exemplo a integração à arquitetura.

Abordando esta integração, existem dois tipos principais, os Building Integrated Photovoltaics (BIPV) e os Building Applied Photovoltaics (BAPV). Os BIPVs são relacionados à integração fotovoltaica pensada na fase do projeto da construção e considerando todos os aspectos da tecnologia em concordância com os outros materiais utilizados no projeto. Já os BAPVs são elementos fotovoltaicos aplicados em construções já existentes, ou seja, um projeto fotovoltaico feito posteriormente a construção do edifício. A esse respeito, Cronenberger et al. (2014 p. 45) e Santos (2013 p.31) apontam que, em projetos BIPV, os elementos fotovoltaicos da construção fazem parte do envelope do edifício, ou seja, a integração fotovoltaica elaborada durante o projeto da construção. Já os elementos BAPVs não substituem ou atuam como componente da construção, mas adicionam e adaptam elementos fotovoltaicos a algo já construído.

Ao utilizar a energia solar por meio da conversão fotovoltaica para suprir a necessidade energética de diversos produtos é possível pensar nos benefícios decorrentes, que podem incluir a utilização fora da rede convencional de energia, a recarga de baterias e a ampliação da autonomia dos produtos. Este fato é reafirmado por Alsema et al. (2005) que sinalizam como vantagens a operação de produtos portáteis de forma prolongada ou totalmente independentes da rede de energia, a redução do consumo e do desgaste de baterias e a conscientização da sociedade de que a energia solar fotovoltaica pode ser considerada uma opção viável de energia. Contudo, Alsema et al. (2005) e Reinders e Akkerman (2005) relembram que estas vantagens existem apenas se o design do produto integrar, de forma adequada e com o dimensionamento correto, a tecnologia fotovoltaica.

Para os autores, um bom design combina uma alta confiabilidade da fonte de alimentação, uma interface user-friendly, uma estética atraente e um baixo impacto ambiental. Os autores mencionam a necessidade de se conhecer a performance dos módulos fotovoltaicos em ambientes internos, de acordo com o tipo de produto a ser projetado. Reinders e Akkerman (2005) apontam ainda a necessidade de um sistema fotovoltaico integrado ser projetado como um todo.

Reinders et al. (2009, p. 3183) apontam que aspectos do design que podem melhorar o apelo de um produto são a usabilidade, a interatividade, a segurança e a personalização. Além disso, os autores comentam que um fornecimento autônomo de energia pode levar a uma maior interação entre o usuário e o produto e também a uma maior liberdade do uso espacial.

O Quadro 1 compara processos de design de produtos PIPV descritos por Alsema et al. (2005) e Reinders et al. (2009). 


\begin{tabular}{|c|c|}
\hline Alsema et al. (2005) & Reinders et al. (2009) \\
\hline Geração de alternativas & Identificação da tarefa \\
\hline Avaliação de alternativas & Especificação da tarefa \\
\hline Seleção da alternativa a ser produzida & Geração do conceito \\
\hline Projeto detalhado do protótipo & Criação de layout preliminar \\
\hline Desenvolvimento do protótipo & Criação de layout definitivo \\
\hline Teste do protótipo & Documentação do processo e do projeto \\
\hline Desenvolvimento do projeto final & Apresentação da solução \\
\hline Desenvolvimento do produto final & \\
\hline
\end{tabular}

Quadro 1 - Processos de design de produtos PIPV

No que tange o design de módulos fotovoltaicos, é possível considerar alguns aspectos do design modular, que incluem a facilidade de produção e montagem, a flexibilidade, e a redução no custo de produção proveniente da padronização. Contudo, como apontam Adamovic et al. (2017, p.2), os móduos fotovoltaicos convencionais, de produção em massa, em muitos casos não são adequados para a aplicação em projetos BIPV, por exemplo em fachadas e superfícies.

Algumas empresas apresentam estruturas para a fixação dos módulos tradicionais de silício de forma integrada à arquitetura, contudo a estética apresentada por estes módulos é bastante específica. Neste ponto, Adamovic et al. (2017 p. 3-4) comentam que o ideal é que os módulos fotovoltaicos a serem utilizados em projetos BIPV sejam passiveis de mudanças em suas formas, tamanhos, cores, transparências e especificidades elétricas. Tal fator pode ser atingido com a possibilidade de personalização dos módulos, já concedidas, por exemplo, por empresas que trabalham com módulos de filme fino.

Como forma de englobar o desenvolvimento de novos módulos para projetos BIPV, processos de design de produtos, como os apresentados na tabela 1 deste estudo podem ser utilizados. Neste cenário há uma substituição de peças construtivas tradicionais cujo avanço pode ser observado notavelmente em países como Alemanha e Suíça, onde já são encontrados diversos exemplos de edifícios BIPV. Um exemplo de BIPV pode ser observado na Figura 4. 


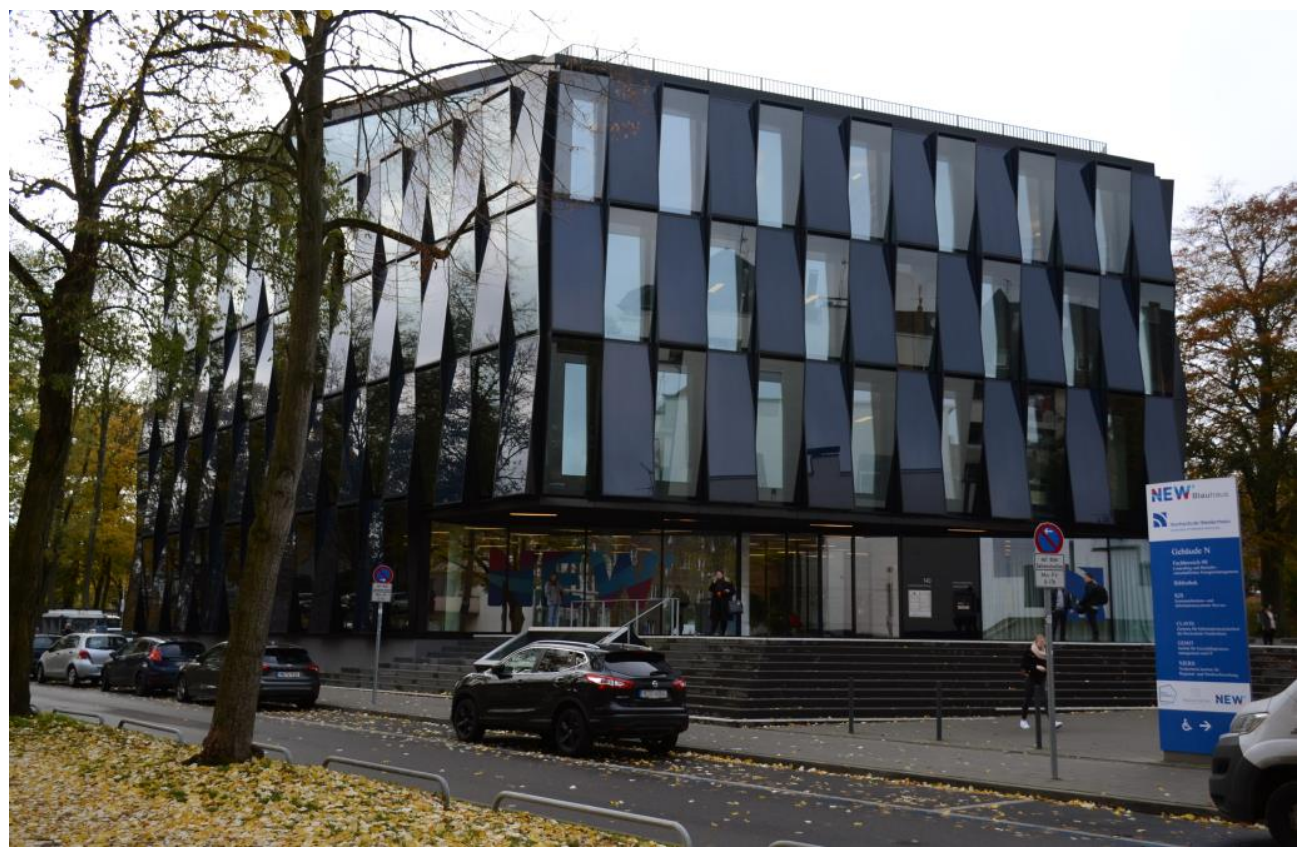

Figura 4 - NEW-Blauhaus Fonte: fotografia - Silveira, F. A. (2020) edifício - Hochschule Niederrhein. Projeto - kadawittfeldarchitektur

A imagem refere-se à NEW-Blauhaus, um edifício central no campus da Universidade Hochschule Niederrhein localizada em Monchengladbach na Alemanha. O projeto deste edifício foi realizado pelo escritório kadawittfeldarchitektur da cidade de Aachen, também na Alemanha. Neste exemplo de BIPV é possível observar a utilização de módulos fotovoltaicos em conjunto com janelas de vidro para compor a pele do edifício.

\section{Método de pesquisa}

O método Design Science Research é utilizado a partir do modelo de Dresch et al. (2015), segundo o qual o problema é identificado; posteriormente, há a conscientização deste problema; faz-se a revisão bibliográfica sistemática (RBS); na sequência o artefato para a solução do problema e as classes de problemas são identificados. Em seguida, o artefato é proposto, desenvolvido, avaliado, as aprendizagens são explicadas e a conclusão é realizada de modo a garantir a validade da pesquisa e demonstrar as decisões e limitações encontradas. Na sequência a solução encontrada por meio do artefato é generalizada para a classe de problemas e os resultados são divulgados.

No presente artigo são abordados os pontos até o desenvolvimento do artefato. A avaliação, a aprendizagem e a conclusão serão abordadas em estudos futuros.

\section{Identificação do artefato e classes de problemas}

As lacunas que justificam estudo, como já apresentadas anteriormente, têm relação como modo com que os projetos são realizados, as competências necessárias para arquitetos, designers e funcionários de montagem e manutenção, e a transmissão do conhecimento para a realização destes projetos. 
Com base nas informações relacionadas às classes de problemas, observou-se a necessidade da construção de um artefato capaz de demonstrar a variação da energia elétrica produzida anualmente, num dado local com seu regime típico de insolação, por módulos fotovoltaicos em diversas inclinações e orientações, sendo estes pertencentes às três gerações de células fotovoltaicas abordadas neste estudo.

Existem atualmente alguns dispositivos que realizam a medição da irradiação solar, como os piranômetros. O Instituto de Tecnologia do Paraná (TECPAR) também realiza medições de dados de irradiação em sua estação solarimétrica que é integrada à rede nacional do Sistema de Organização Nacional de Dados Ambientais (Sonda), do Instituto Nacional de Pesquisas Espaciais (Inpe). Contudo, as medições realizadas pelos piranômetros e pelo TECPAR não abrangem o objetivo deste trabalho, pois não realizam a medição segregada por orientação e inclinação, bem como não utilizam módulos fotovoltaicos comerciais.

Além dos dispositivos que realizam as medições mencionados anteriormente, existem grupos de pesquisa que realizam simulações da irradiação solar para a conversão fotovoltaica, a exemplo do trabalho de Santos (2013) cuja simulação para a cidade de Curitiba - Paraná pode ser observada na Figura 5. Estas simulações abrangem grande parte do território nacional. A pesquisa abordada neste estudo difere das simulações realizadas pelo fato de buscar dados reais para corroborar, ou refutar os resultados encontrados em simulações.

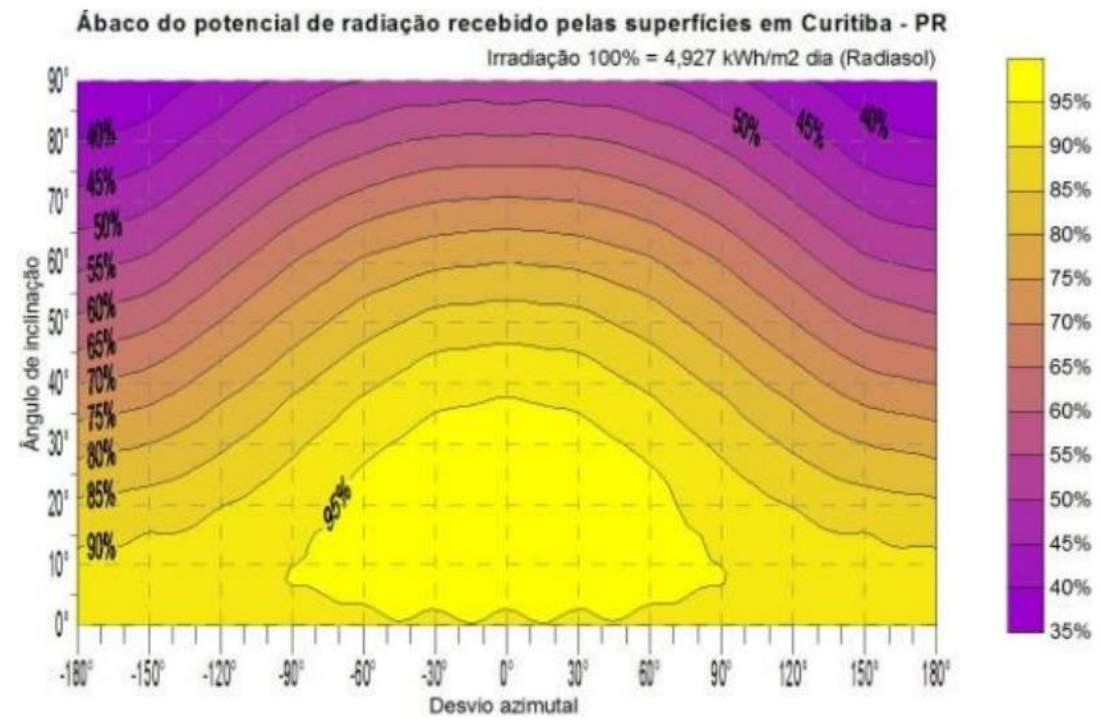

Figura 5 - Abaco de Curitiba - PR. Fonte: Santos (2013 p. 149)

$\mathrm{Na}$ imagem as cores mais claras indicam os índices mais altos de irradiação solar $\left(\mathrm{kWh} / \mathrm{m}^{2}\right.$ ano) ao longo do ano, e nas cores mais escuras as menores incidências de irradiação.

\section{Proposição do artefato}

O artefato proposto realiza a medição de radiação solar em diversas condições de orientação e inclinação - a maior parte sub-ótimas - e utiliza tecnologias de conversão fotovoltaica disponíveis no mercado. Para a conversão são utilizados módulos compostos por células de 
silício policristalino, silício amorfo e módulos orgânicos - OPV. Na Tabela 1 é possível ver as características dos módulos utilizados no desenvolvimento do artefato.

\begin{tabular}{|c|c|c|c|}
\hline Módulo & SinoSola SA10-36P & $\begin{array}{c}40 \% \text { Transparent Solar } \\
\text { Panel - SolarFirst }\end{array}$ & $\begin{array}{c}\text { InfinityPV foil - OPV } \\
\text { demonstrator }\end{array}$ \\
\hline Tecnologia & Silício Policristalino & Silício Amorfo & OPV* \\
\hline Potência Máxima & $10 \mathrm{~W}$ & $5,2 \mathrm{~W}$ & $3 \mathrm{~W} / \mathrm{m}$ \\
\hline $\begin{array}{c}\text { Tensão em } \\
\text { circuito aberto }\end{array}$ & $22,5 \mathrm{~V}$ & $31,9 \mathrm{~V}$ & - \\
\hline $\begin{array}{c}\text { v em curto } \\
\text { circuito }\end{array}$ & $0,6 \mathrm{~A}$ & $0,23 \mathrm{~A}$ & - \\
\hline $\begin{array}{c}\text { Tensão máxima } \\
\text { Corrente máxima }\end{array}$ & $19,0 \mathrm{~V}$ & $25,3 \mathrm{~V}$ & $0,04 \mathrm{~A}$ \\
\hline $\begin{array}{c}\text { Dimensões } \\
0,6 \mathrm{~A}\end{array}$ & $\begin{array}{c}350 \mathrm{~mm} \times 252 \mathrm{~mm} \times \\
25 \mathrm{~mm}\end{array}$ & $\begin{array}{c}350 \mathrm{~mm} \times 252 \mathrm{~mm} \times \\
6 \mathrm{~mm}\end{array}$ & $140 \mathrm{~mm} \times 100 \mathrm{~mm}$ \\
\hline
\end{tabular}

Tabela 1 - Comparação de módulos do artefato. *Por unidade de comprimento da fita.

$\mathrm{O}$ artefato segue alguns pré-requisitos como a disposição alternada das três tecnologias de conversão em azimute e inclinação variados, a utilização de um valor controlado de albedo (fração da radiação incidente numa superfície que é refletida de modo difuso), o afastamento do solo no intuito de reduzir o sombreamento projetado pelos edifícios próximos e a possibilidade de uso do artefato como espaço de vivência pelos alunos da Universidade Federal do Paraná.

\section{Desenvolvimento do Artefato Zero}

No início do processo do desenvolvimento do artefato foi necessário buscar por fornecedores de módulos fotovoltaicos policristalinos, de filme fino e OPV com dimensões similares. Nesta etapa, encontramos alguns contratempos como falta de fornecedores no Brasil para os módulos de filme fino e a quantidade reduzida de fornecedores de OPV no mundo.

Após a definição de compra dos três modelos de módulos, foram considerados para o desenvolvimento do artefato o espaço disponível para a inserção do dispositivo de medição, os tipos de módulos fotovoltaicos, a necessidade do controle do albedo e a disposição dos módulos em relação ao azimute e à inclinação. A partir destas informações, foi definida a quantidade necessária de módulos e como estes seriam dispostos. Para viabilizar o projeto, foram selecionados seis ângulos de inclinação entre $7,5^{\circ}$ e 82,5 e utilizados 124 módulos em um domo com diâmetro de 1,45 m, como pode ser observado na Figura 6. 


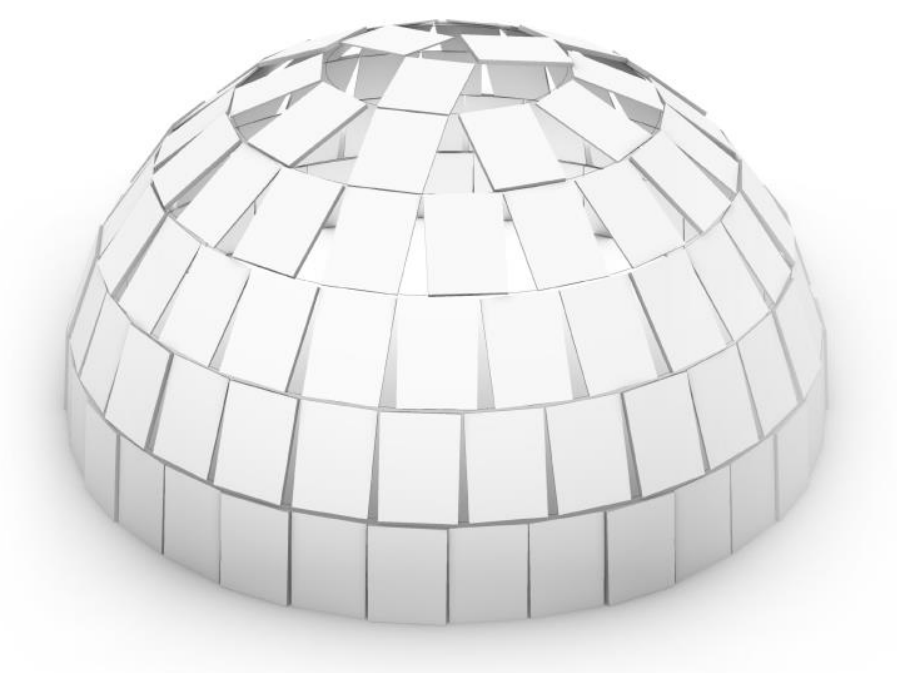

Figura 6 - Disposição dos módulos no domo. Fonte: Os autores

Com a definição das dimensões e da disposição dos módulos do domo (de forma alternada e em concordância com a figura acima) foi possível iniciar o planejamento da base do artefato. Para isso, foram definidos alguns requisitos com base na revisão teórica e em conceitos de projetos de design e arquitetura em conjunto com a equipe do projeto. Tais requisitos são:

- Estrutura com uso reduzido de materiais e com capacidade de suportar tanto o domo como a superfície rebatedora;

- Superfície rebatedora inclinada e com formato definido a partir do domo para igualar sua interferência nos módulos do entorno do domo (padronização do albedo).

- Proteção do equipamento;

- Artefato como espaço para socialização dos estudantes;

- Altura elevada para evitar sombreamento proveniente de árvores e outros edifícios entre $07 \mathrm{~h}$ e $17 \mathrm{~h}$.

- Resistência ao vento

Após a definição dos requisitos iniciou-se a geração de alternativas. Visando à redução do vandalismo, optou-se por aproximar o equipamento do público, antes que afastá-lo. Por isto, foram tomadas por base construções conhecidas como coretos (coberturas situadas ao ar livre, para abrigar bandas musicais em concertos, festas e romarias) dado seu uso similar ao objetivo de socialização dos estudantes. Alguns exemplos da primeira geração de alternativas podem ser observados na Figura 7. 

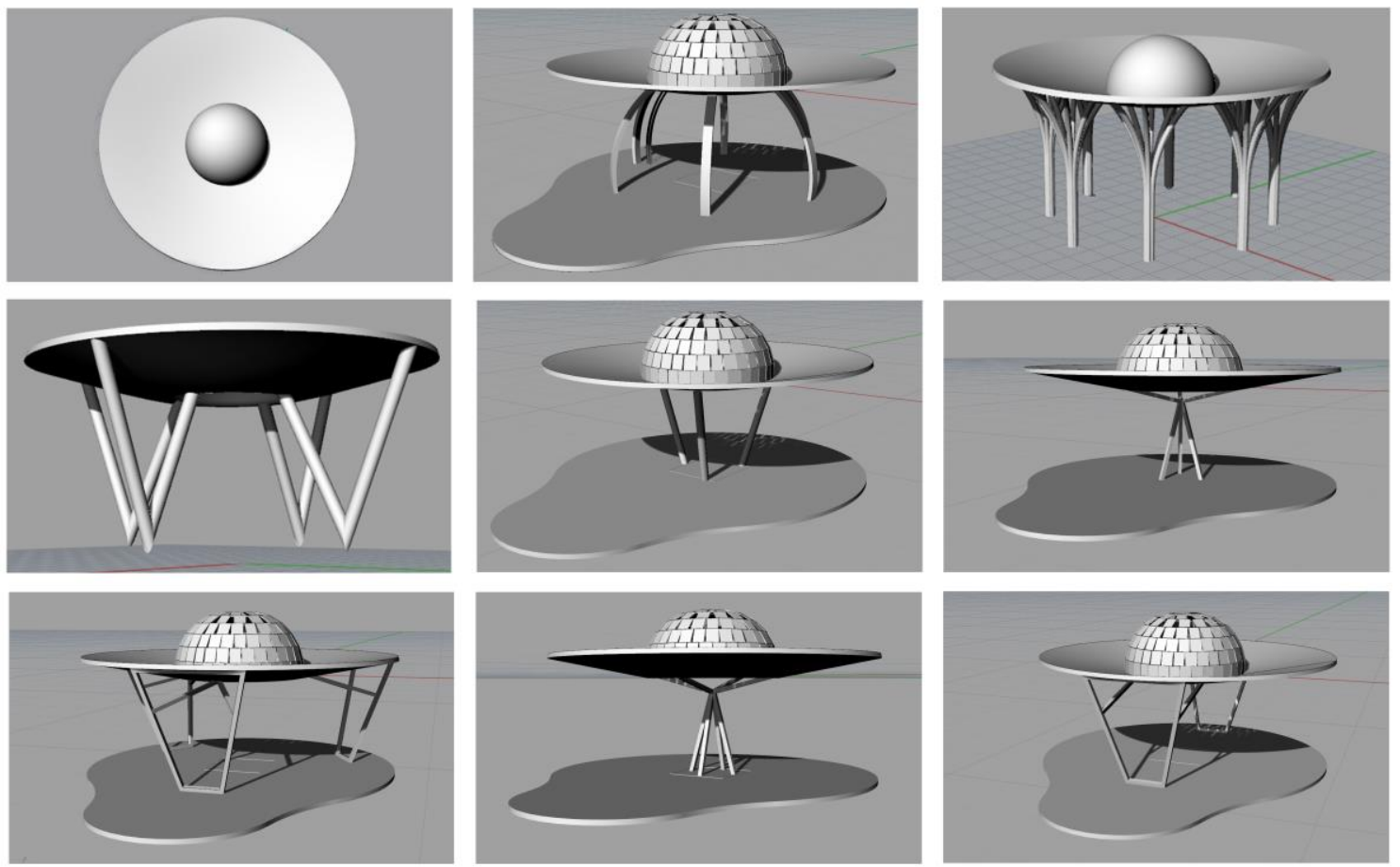

Figura 7 - Geração de alternativas inicial Fonte: Os autores

Nesta geração de alternativas tomou-se como ponto principal o domo, como abordado anteriormente e a superfície rebatedora. Estes definiram as dimensões finais do artefato e podem ser visualizados na linha superior à esquerda. Com estes pontos definidos, iniciou-se a busca por uma base adequada ao projeto e que respeitasse os requisitos apresentados.

A partir das alternativas geradas inicialmente e reuniões entre os integrantes do projeto, optou-se por buscar auxílio para questões técnicas relacionadas à estrutura, como meios viáveis para a produção do artefato em metal e simulações da força exercida pelo vento. Nesta etapa foram consultadas empresas de estruturas metálicas e o Departamento de Engenharia Mecânica da Universidade. Após esta etapa foi realizada uma nova geração de alternativas, exemplificada na Figura 8.
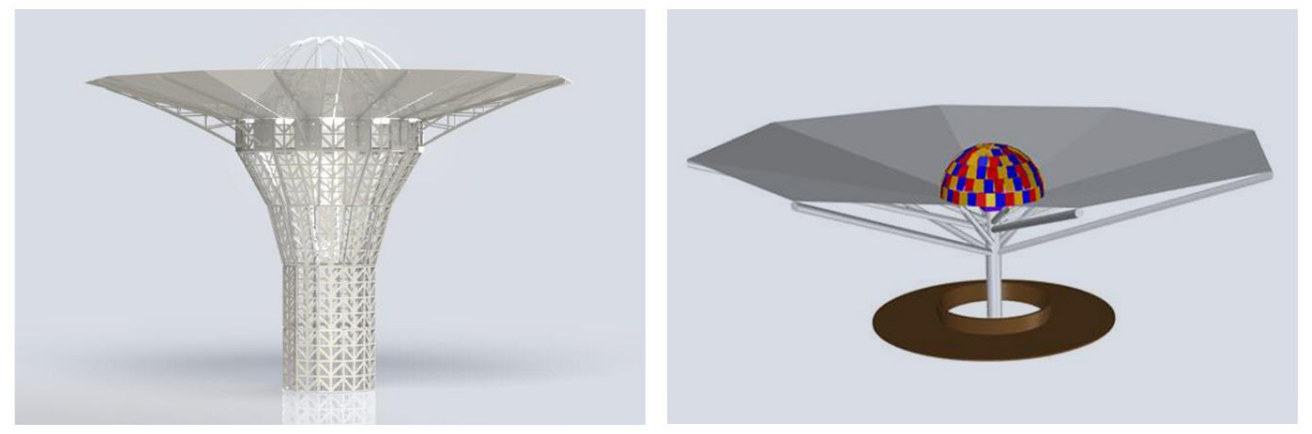

Figura 8 - Exemplos da segunda geração de alternativas. Fonte: Os autores (2019)

Com a escolha da alternativa mais adequada ao projeto, considerando o uso de materiais e os requisitos, foi realizada uma simulação da resistência do artefato ao vento. Esta simulação foi 
realizada no software ANSYS e considerou as características de vento da cidade de Curitiba Paraná, parte fa simulação pode ser observada na Figura 9 e na Figura 10.

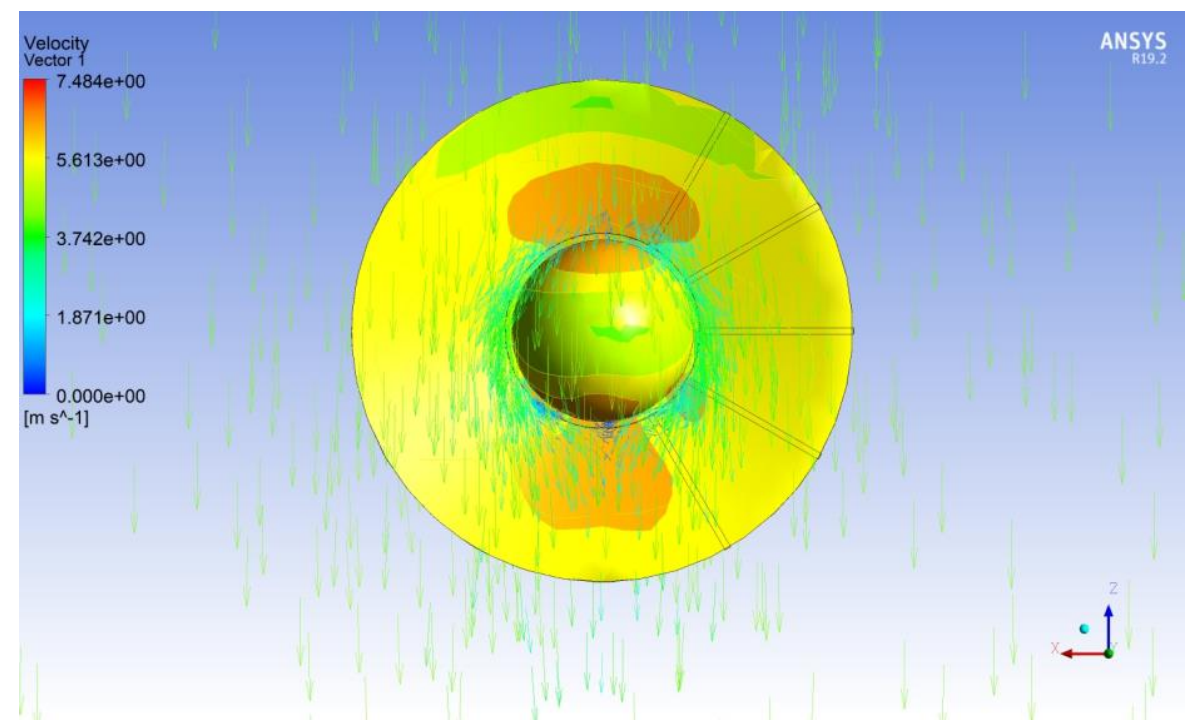

Figura 9 - Simulação ventos Fonte: Strobel (2019)

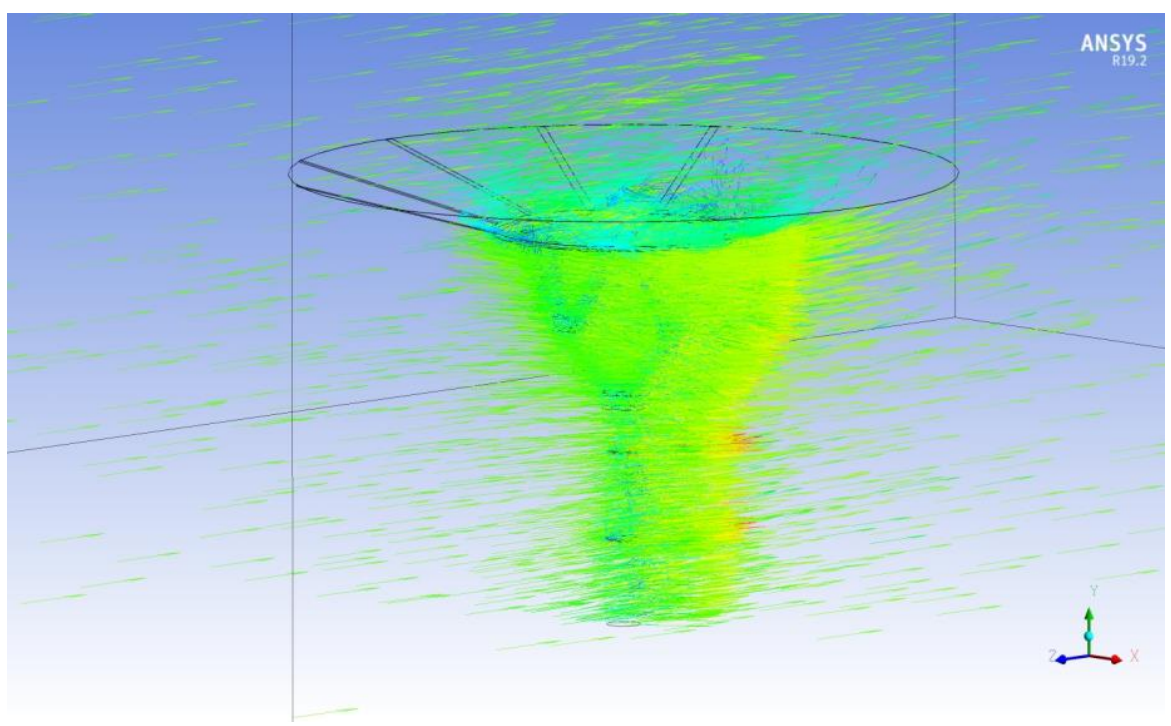

Figura 10 - Simulação ventos Fonte: Strobel (2019)

Após a primeira simulação foram feitos ajustes na estrutura para melhorar sua resistência ao vento e promover segurança aos componentes do artefato, bem como a comunidade da universidade.

Com a finalização dos ajustes e o desenvolvimento do modelo final do artefato, tornou-se possível estipular as dimensões finais de diâmetro $(8 \mathrm{~m})$ e altura $(6 \mathrm{~m})$, bem como calcular o peso, a fundação do artefato e iniciar a busca por fornecedores de estruturas metálicas, lonas para a superfície rebatedora e engenharia de hardware para o dispositivo de medição. 
Quanto ao dispositivo de medição, a produção de dados do artefato será realizada utilizando equipamentos de telemetria e tendo como fonte os 124 módulos fotovoltaicos do projeto, com a produção de energia elétrica mês a mês para cada orientação.

\section{Resultados preliminares}

Numa etapa inicial, foi feita por Bonetto (2019) a modelagem e simulação detalhada em planilha eletrônica de cálculos, estimando em termos bastante precisos a contribuição da radiação direta e da radiação difusa sobre cada módulo do artefato. Com relação à radiação refletida, foi estimado um valor inicial de caráter conservador, pois não inclui todos os caminhos dos raios de luz.

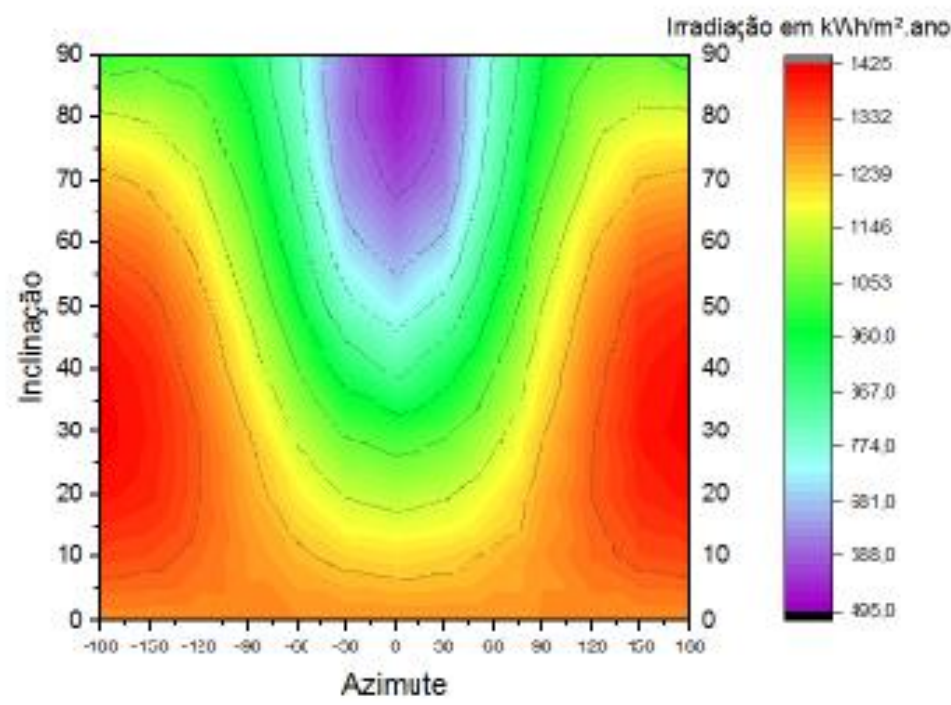

Figura 11 - Combinação das orientações e inclinações do PV formando um média anual da irradiação global em kWh/m².ano. Fonte: Bonetto (2019)

A Figura 11 traz um gráfico da irradiação global em $\mathrm{kWh} / \mathrm{m}^{2}$ ano. Nela já se percebe a influência decisiva do albedo em permitir que a pior combinação de orientação (sul) e inclinação $\left(90^{\circ}\right)$ gere $35 \%$ de conversão em relação à melhor combinação (respectivamente, norte e $\left.25^{\circ}\right)$.

A medição resultará em diagramas como demonstrado na Figura 11, referente a uma simulação do mesmo domo realizada, no âmbito deste projeto, para a cidade de Curitiba Paraná.

Este diagrama deve ser confirmado pelo artefato em funcionamento. Para diferentes valores de inclinação e azimute, será conhecido o valor esperado de irradiação. Desta forma, o desempenho de projetos de sistemas fotovoltaicos montados fora do par ideal de orientação e inclinação (condições sub-ótimas) poderão ser precisamente quantificados. Esperamos que o montante perdido em termos de produção de energia elétrica seja compensado pelo custo reduzido ao se integrar o sistema fotovoltaico na edificação, evitando redundância de materiais. 
Ressalte-se que somente com a simulação já foi possível inferir que os módulos fotovoltaicos, mesmo em orientações diferentes daquela tida como ideal, convertem uma quantidade signficativa de energia solar. Nestes casos, o albedo ganha maior importância, pois a irradiação refletida pela sua superfície pode compensar, em relação à geração de energia, a diferença na orientação e na inclinação dos módulos, principalmente nos casos em que eles se apresentam em posições mais verticais. A decisão sobre cores claras para telhas, calçadas e outras superfícies pode ter impacto decisivo no desempenho dos sistemas BIPV.

\section{Considerações finais}

A aplicação de dispositivos fotovoltaicos em projetos BIPV ou PIPV no contexto brasileiro ainda enfrenta certa resistência, seja pelo desconhecimento na elaboração de projetos ou pela ausência de bons exemplos em território nacional. Como uma forma de auxiliar no desenvolvimento desta área, este estudo compreende as implicações ao desviar superfícies captadoras da orientação tida como ideal e busca apresentar meios que possam atenuar o impacto da redução da conversão fotovoltaica da energia solar em condições energeticamente sub-ótimas. Um exemplo é autilização do albedo como forma de complementar a radiação solar incidente nos módulos em um projeto BIPV.

O artefato apresentado neste artigo utiliza módulos comerciais e realiza medições segregadas por orientação e inclinação. Este artefato está em fase de construção e conta com uma estrutura metálica, domo com 124 módulos fotovoltaicos e o albedo composto por lonas com índice de reflexão conhecido. Os dados de irradiação apresentados após a coleta de dados serão analisados e disponibilizados futuramente por meio de um guia de dimensionamento fotovoltaico.

Trabalhos futuros podem apresentar a conclusão da construção do artefato, os testes na coleta de dados e ampliação no tempo de medição para acompanhar fenômenos climáticos, bem como alterações no albedo para maior índice de refletância e albedo nulo.

\section{Referências}

Alsema, E.A.; Elzen, B.; Reich, N.H.; van Sark, W.G.J.H.M.; Kan, S.Y.; Silvester, S.; Veefkind, M.; Jelsma, J. Towards an optimized design method for pv-powered consumer and professional applications - the syn-energy project. Proceedings of the 20th European Photovoltaic Solar Energy Conference and Exhibition. (3-936338-19-1) p. 1981-1984, Barcelona, 2005.

ASSOCIAÇÃO BRASILEIRA DE NORMAS TÉCNICAS - NBR 10899 Energia solar fotovoltaica - Terminologia. Rio de Janeiro 2013.Cronemberger, J. Corpas, M. A. Isabel Cerón, I. Caamaño-Martín, E. Sánchez, S. V. BIPV technology application: Highlighting advances, tendencies and solutions through Solar Decathlon Europe houses. Energy and Buildings 83 p. 44-56. 2014. Disponível em: https://www.sciencedirect.com/science/article/ pii/S0378778814003491?casa token=kByw8RSOo14AAAAA:yS4F5D5dFNL4J8uv9qWXd H30Szw3CrvG7VOYa-_jepCxIF79wIAMaee9wedFjOVXRLvCJ9yAsXOz Acesso em 27 abril 2020 
BONETTO, M. Simulação numérica da radiação total em um artefato de coleta de dados na UFPR (campus politécnico). Trabalho de conclusão de curso em engenharia ambiental. Orientadores: Marcelo Risso Errera e Aloísio Leoni Schmid. UFPR: 2018.

DRESCH, A; LACERDA, D. P; ANTUNES JR, J. A. V. Design Science Research: método de pesquisa para avanço da ciência e tecnologia. Bookman, 2015

FROTA, A. B.. Manual de Conforto Térmico: Arquitetura, urbanismo. Nobel, 2001.

HANGZHOU AMPLESUN SOLAR TECHNOLOGY. 110W double glass amorphous silicon thin film. Fotografia. Disponível em: https://amplesun.en.ecplaza.net/ Acesso em: 15 abril 2020

INFINITY PV. Organic solar cells. Fotografia. Disponível em: https://infinitypv.com/technology/opv. Acesso em: 15 abril 2020.

MACHADO, C.T.; MIRANDA, F.S. Energia Solar Fotovoltaica: Uma Breve Revisão. Revista Virtual de Química. [Online]. 7, (1984-6835), 126-143. 2014.

QUERINO, C. A. S. Avaliação da radiação solar em ecossistema de manguezal tropical em Alagoas, Brasil. Dissertação (mestrado em Meteorologia: processos de superfície terrestre) - Universidade Federal de Alagoas Maceió, 297p. 2006.

REINDERS A; AKKERMAN R. Design, production and materials of PV powered consumer products - the case of mass production. Proceedings of the 20th European Photovoltaic Solar Energy Conference and Exhibition. (3-936338-19-1) p. 1981-1984, Barcelona, 2005.

REINDERS A.; BOER A.; WINTER A.; HAVERLAG M. Designing PV powered led products - integration of PV technology in innovative products. Proceedings of 24th EU Photovoltaic Solar Energy Conference and Exhibition. (3-936338-25-6) p. 3179-3183, Munich, 2009.

SANTOS, I. P. Desenvolvimento De Ferramenta De Apoio À Decisão Em Projetos De Integração Solar Fotovoltaica à Arquitetura. Tese (Doutorado em Engenharia Civil) Universidade Federal de Santa Catarina. Florianópolis, 278p., 2013.

SLAOUI, A. Inorganic materials for photovoltaics: Status and futures challenges. In: EPJ Web of Conferences, 5th course of the MRS-EMRS Materials for Energy and Sustainability and 3rd course of the EPS-SIF International School on Energy. 148. 2017. Disponível em: https://www.epj-conferences.org/articles/epjconf/abs/2017/17/epjconf_epssif2017_00007/

epjconf_eps-sif2017_00007.html Acesso em 25 out 2019.

STROBEL, C, S. Simulação da ação do vento no artefato. Curitiba, 2019.

SUNFLOWER SOLAR WATER HEATER Quelle est la différence entre polycristallin monocristallin et thyn film. Fotografia. Disponível em . Acesso em: 15 abril 2020.

VILLALVA, M. G; GAZOLI, J. R. Energia solar fotovoltaica: conceitos e aplicações. São Paulo: Ed. Ética Ltda. 2013. Vol 1. 224p.

YAMAMOTO, N. A. D. Células Solares Orgânicas Baseadas nas Estruturas Heterojunção em Bicamada e Heterojunção de Volume Tese (Doutorado em Física) Universidade Federal do Paraná. Curitiba, 133p., 2014.

\section{Sobre os autores}

Flávia Aparecida Silveira - Designer, especialista em gestão ambiental e desenvolvimento sustentável. Estudante de mestrado em design no Programa de Pós Graduação em Design da 
UFPR - PPGDesign. Pesquisa a energia fotovoltaica fora das condições ideais de orientação e inclinação.

ORCID. https://orcid.org/0000-0002-4097-7073

Aloísio Leoni Schmid - Engenheiro mecânico, mestre e doutor, tendo sido orientado em Karlsruhe pelo Prof. Dr. Jürgen Schmid, pioneiro da integração de sistemas fotovoltaicos à arquitetura. Na UFPR, pesquisa o ambiente construído, desde aspectos qualitativos até o desempenho, desenvolvendo software de análise. Participa dos Programas de Pós-Graduação em Design e em Engenharia Civil, além dos cursos de graduação de Arquitetura e Urbanismo, e de Tecnologia em Luteria.

ORCID. https://orcid.org/0000-0003-2737-6246 